G. Jones, C. Lovell, H. Morgan, and K.-P. Zauner. Organising Chemical Reaction Networks in Space and Time with Microfluidics. International Journal of Nanotechnology and Molecular Computation (IJNMC) 3(1):35-56, 2011. doi:10.4018/jnmc.2011010104

\title{
Organising Chemical Reaction Networks in Space and Time with Microfluidics
}

\author{
Gareth Jones \& Chris Lovell \& Hywel Morgan \& Klaus-Peter \\ Zauner \\ Science and Engineering of Natural Systems \\ School of Electronics and Computer Science \\ University of Southampton \\ UK, SO17 1BJ
}

\begin{abstract}
Information processing is essential for any lifeform to maintain its organisation despite continuous entropic disturbance. Macromolecules provide the ubiquitous underlying substrate on which nature implements information processing and have also come into focus for technical applications. There are two distinct approaches to the use of molecules for computing. Molecules can be employed to mimic the logic switches of conventional computers or they can be used in a way that exploits the complex functionality offered by a molecular computing substrate. Prerequisite to the latter is a mapping of input-output transform provided by the substrate. In the present paper we review microfluidic technology as a versatile means to achieve this, show how we use it, and provide proven recipes for its application.
\end{abstract}

\section{Introduction}

Much of the information processing within biological systems relies on the interactions of macromolecules, particular proteins, with one another and with their environment (Bray, 1995). Recognising this, enzymes have been applied in the implementation of Boolean logic gates, as demonstrated by Conrad and Zauner (Zauner \& Conrad, 2001), and by Sivan et. al. (Sivan, Tuchman, \& Lotan, 2003). Willner's group extended the principle to networks of

Gareth Jones, School of Electronics and Computer Science, University of Southampton; Chris Lovell, School of Electronics and Computer Science, University of Southampton; Hywel Morgan, School of Electronics and Computer Science, University of Southampton; Klaus-Peter Zauner, School of Electronics and Computer Science, University of Southampton. The reported work was supported in part by a Microsoft Research Faculty Fellowship to Klaus-Peter Zauner. Correspondence concerning this article should be addressed to Klaus-Peter Zauner, School of Electronics and Computer Science, University of Southampton, UK, SO17 1BJ. Email: kpz@ecs.soton.ac.uk 
enzymes forming single logic gates (Baron, Lioubashevski, Katz, Niazov, \& Willner, 2006c), concatenated logic gates (Niazov, Baron, Katz, Lioubashevski, \& Willner, 2006) and enzymes acting in parallel as half-adders and half-subtracters (Baron, Lioubashevski, Katz, Niazov, \& Willner, 2006a, 2006b). More recently, Katz and coworkers have shown many iterations of enzymatic logic gates and also interfaced them to conventional electronics (Pita et al., 2009; Zhou et al., 2009).

Given the structural complexity of proteins and their broad range of functionality in nature, the information processing available through individual enzymes is unlikely to be limited to Boolean logic. A more sophisticated application of proteins in molecular information processing, however, faces the obstacle that there is as at present no method for designing proteins for purpose available. Instead of engineering enzymes for a particular application, through characterising the response behaviour of enzymes available from nature, arguably new modes of information processing could be supported ultimately facilitating the application of enzymatic computers (Zauner \& Conrad, 2001). Fully surveying the response characteristics of an enzyme, in particular in a technical setting not limited to physiological conditions, requires sampling over high-dimensional parameter spaces. Time and materials are typically very limited compared to the large parameter space preventing a detailed investigation of behaviour. To make the best use of available resources we use autonomous experimentation based on a physical platform that minimises resource requirements per experiment with machine learning techniques to drive the experimentation platform.

Microfluidic technology provides a means of significantly reducing the resource requirements per experiment when compared to conventional laboratory hardware. Microfluidic devices may be computer-controlled through the inclusion of on-chip valves thus allowing for complex protocols to be performed. As a technology microfluidics is of more general interest for the study and implementation of molecular level information processing than in the context of our application. For the benefit of the readers not familiar with its possibilities we review key concepts of microfluidics in Section .

For our purpose of developing a microfluidic experimentation platform particularly to characterise enzymes for information processing we have specific requirements. Ideally many different chemicals would be used to probe the behaviour of an enzyme. This necessitates a complex microfluidic device containing many channels with associated pumps and valves. Thus a low-cost implementation is strongly desired. In addressing those requirements we developed a low-cost computer interface (cf. Section ) and fabrication protocols that do not require sophisticated equipment or a cleanroom environment. For the benefit of readers who contemplate the use of microfluidic architectures we provide the details of the fabrication protocols in the Appendices. The fabrication protocols and the new interface technique should make it feasible to probe networks of enzymes with multiple chemical signals and therefore open up the potential for more complex enzymatic computing to be performed.

\section{Microfluidics}

Microfluidics is implemented through the patterning of micrometre-sized networks of fluidic channels on a substrate material. The fluidic channels may be designed such that multiple laboratory functions, mixing, diluting and measurement are integrated into a chip no larger than a few centimetres square - giving rise to the term lab-on-a-chip. As a technol- 
ogy it allows for a more fine-grained control over reaction condition than what is typically available in bulk chemistry. This is largely due to the fact that in small volumes surface effects are more prominent.

\section{Laminar Flow}

The behaviour of fluids flowing in microscale channels is markedly different to the familiar behaviour of fluids at the macroscale. At the macroscale, fluids tend to flow turbulently and mix readily whereas microscale flows do not. This difference arises from a shift in the competition between the inertial and viscous forces that act on a flowing fluid. This competition is represented by the dimensionless Reynolds number $(R e)$ :

$$
R e=\frac{\text { Inertial Forces }}{\text { Viscous Forces }}=\frac{\rho U D_{H}}{\eta}=\frac{U D_{H}}{\nu}=\frac{Q D_{H}}{\nu A}
$$

where $\rho$ is the fluid density, $U$ the mean flow velocity, $\eta$ the absolute (or dynamic) viscosity, $D_{H}$ the characteristic length scale of the system (or hydraulic diameter, equal to $4 \times$ cross-sectional area divided by wetted perimeter), $\nu$ the kinematic viscosity, $Q$ the volumetric flow rate and $A$ the cross-sectional area.

As can be seen from Equation 1, decreasing channel dimensions or reducing the average flow velocity sufficiently will result in viscous forces dominating. This leads to a flow characterised by smooth linear streamlines or laminae and is therefore referred to as 'laminar flow'. Despite the presence of non-linearities in flow from $\operatorname{Re}>=1$, laminar flow is normally indicated by Reynolds numbers of less than 2300 (Beebe, Mensing, \& Walker, 2002). In microfluidic devices laminar flow is typical, which as we shall see next can be particularly helpful.

\section{Dilution and Chemical Gradient Production from Laminar Flow}

Because of the linear and predictable nature of laminar flow, under particular conditions it has been possible in the design process to treat microfluidic networks as analogous to electrical resistive networks. This in turn has allowed for the creation of microfluidic devices containing passive fluidic networks capable of producing chemical concentration gradients (Jeon et al., 2000), linear (Walker, Monteiro-Riviere, Rouse, \& O'Neill, 2007) and/or logarithmic (L. Kim, Vahey, Lee, \& Voldman, 2006; C. Kim et al., 2008) dilutions. The conditions necessary to obtaining electrically equivalent models of microfluidic channels begin with the Navier-Stokes equations.

The nonlinear partial differential Navier-Stokes equations describe the motion of fluids. In the case of a fluid driven through a microchannel by an externally applied pressure with characteristic laminar flow, the Navier-Stokes equations can be solved to relate the pressuredrop along the channel to the velocity field of the fluid. By integrating the velocity field of the fluid over a cross-section of the channel, the volumetric flow rate $Q$ can be determined. As the flow is laminar, the Hagen-Poisseuille law can then be applied and thus the volumetric flow rate $Q$ can be related to the pressure drop $\Delta P$ by way of a fluidic resistance term $R$ :

$$
\Delta P=Q \cdot R
$$




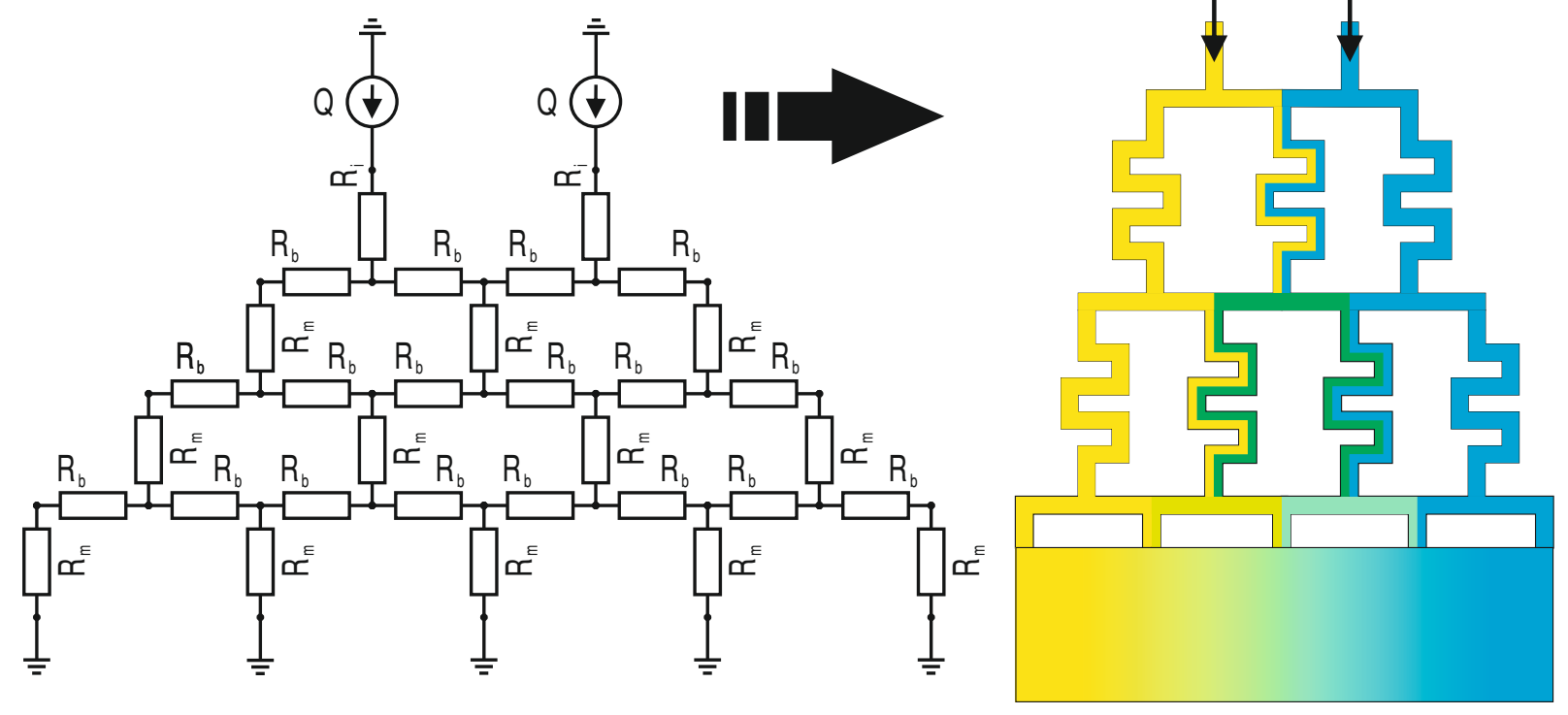

Figure 1. : Chemical gradient generator. Operating within the laminar flow regime, microfluidic channels can be designed analogous to electrical resistive networks leading to the construction of passive devices capable of producing chemical gradients with few liquid inputs. Channel sections are represented by equivalent electrical resistances $R_{i}$ for the inputs, $R_{b}$ for horizontal and $R_{m}$ for the vertical channels. The volumetric flow rate $Q$ is treated analogous to a current source.

which is analogous to Ohm's law. The fluidic resistance for rectangular channels of width $w$, height $h$ and length $L$ can be derived from the analytical solution (Kovacs, 1998):

$$
R=\frac{12 \eta L}{w h^{3}}\left[1-\frac{h}{w}\left(\frac{192}{\pi^{5}} \sum_{n=1,3,5}^{\infty} \frac{1}{n^{5}} \tanh \left(\frac{n \pi w}{2 h}\right)\right)\right]^{-1}
$$

which for square cross-sections of side $a$ approximates to:

$$
R=\frac{28.545 \eta L}{a^{4}}
$$

or rectangular cross-sections with high-aspect ratios $(h \gg w$ or $w \gg h)$ approximates to:

$$
R=\frac{12 \eta L}{w h^{3}}
$$

By fixing the channel width and height, it is then possible to create channels of lengths proportional to their fluidic resistances. Thus as illustrated in Figure 1 networks of varying resistances can be created that divide fluid flow accordingly to allow for in this case gradient 
generation devices. Here we saw how laminar flow can be harnessed to create devices with specific functions, next we consider a hindering effect of laminar flow.

Laminar Flow and Mixing
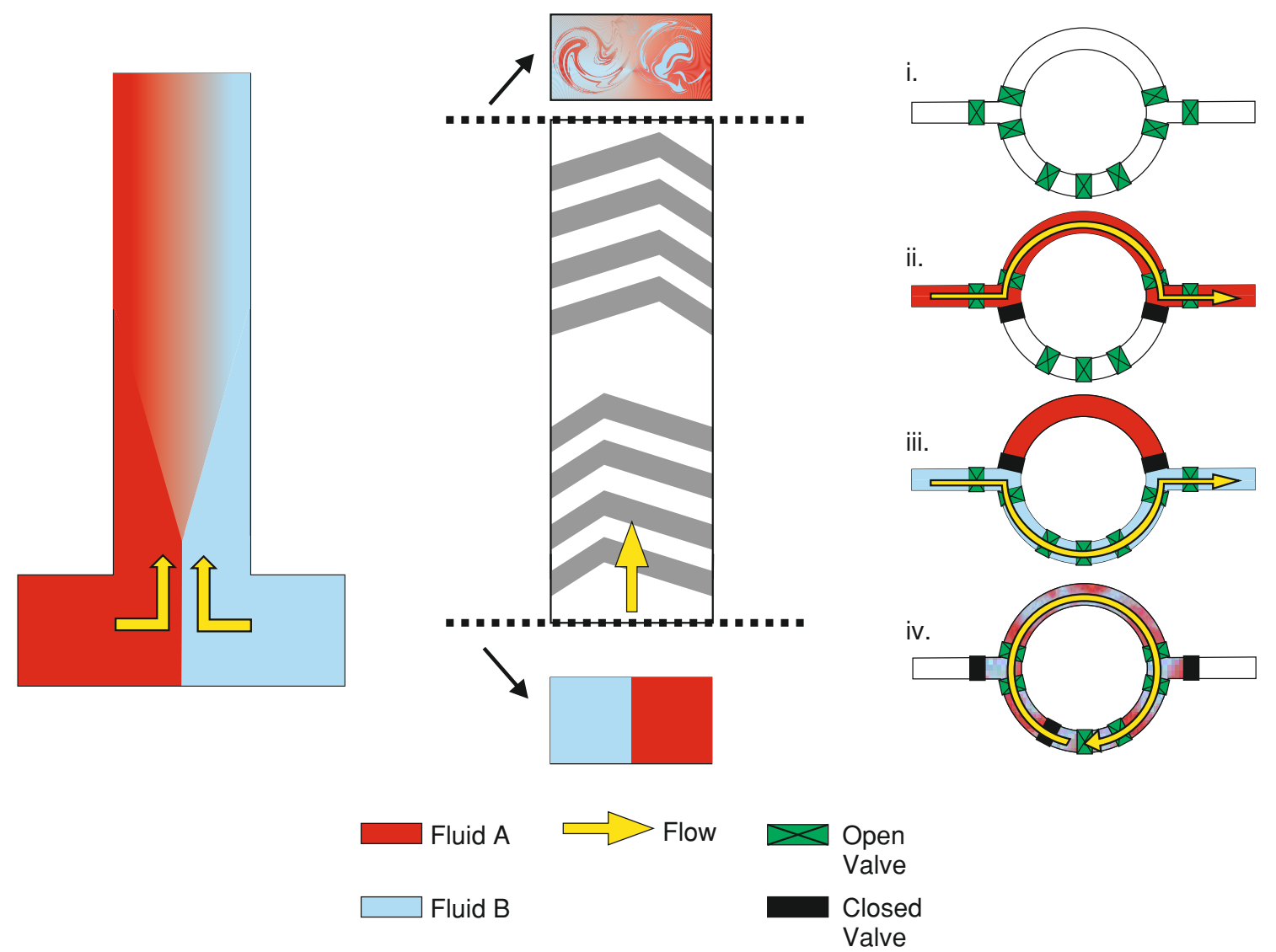

Figure 2. : Mixing in microfluidic devices. In (a) is a T-junction mixer where only axial flow is present and therefore mixing is slow. In (b) is a section of a staggered herringbone mixer. The asymmetrical grooves cause the fluids to be stirred axially as depicted by the cross-section above the dotted line. The operation of a rotary mixer is shown in (c). The mixer is filled with one fluid at a time. The circular channel is then isolated from the inlet and outlet channels. Actuation of the bottom three valves in the circular channel result in mixing of the two fluids.

First we consider some basic concepts of mixing. We start with a square microchannel with sides of length $w$ containing a molecule to be mixed with a characteristic diffusion coefficient $D$. In one dimension, the mixing time is described by the time taken $t$ for the molecule to diffuse the entire width of the channel and is approximated by (Ottino \& Wiggins, 2004):

$$
t \approx \frac{w^{2}}{D}
$$


From Equation 6 the time taken for the molecule to diffuse is dependent on the square power of distance to be travelled. Thus it should come as a surprise that mixing actually tends to take significantly longer in fluids flowing in microchannels. The reason for this is because of laminar flow. At the macroscale the distribution of molecules is subjected to varying pressure and velocity fields, exacerbated when the flow is turbulent. The effect is a continuous reduction of the diffusion distances. In microfluidic devices the laminar flow ensures relatively fixed diffusion distances. Therefore the mixing time is prolonged.

So for example consider the enzyme malate dehydrogenase (MDH) that has a reported diffusion coefficient of $6.45 \times 10^{-7} \mathrm{~cm}^{2} / \mathrm{s}$ (Englard \& Siegel, 1969) or $64.5 \mu \mathrm{m}^{2} / \mathrm{s}$. From Equation 6 to diffuse a microchannel of width $200 \mu \mathrm{m}$ end-to-end (one dimension) would take the enzyme approximately $620 \mathrm{~s}$.

With flow the enzyme will also experience convection in the direction of the flow as well as diffusion across the channel (transport in two dimensions). The convection has little impact on the mixing time but a certain channel length $L$ is required for complete mixing to be achieved. This is approximated from:

$$
L \approx \frac{U w^{2}}{D}
$$

where $U$ is the mean flow velocity. Given the same enzyme and channel width parameters in the example used for Equation 6 and a mean flow velocity $U$ of $100 \mu \mathrm{m} / \mathrm{s}$, from Equation 7 the required channel length is approximated to be $6.2 \mathrm{~cm}$.

An alternate method to estimating the channel length can be found in using the dimensionless Péclet number $(P e)$, which relates the rate of convection of a flow to its rate of diffusion. The Péclet number is defined as:

$$
P e=\frac{U w}{D}
$$

with $\mathrm{Pe}=310$ for the same values used previously. Taking the Pe and multiplying it by the channel width, here $200 \mu \mathrm{m}$, an estimate of the channel length can be obtained, 6.2 $\mathrm{cm}$ as before. Reflecting on Equations and 2-5 it is important to remember that the channel length is proportional to the fluidic resistance. Consequently the channel length required to achieve complete mixing in some cases may impose pressure requirements too large to practically implement. Thus we now consider methods of improving mixing in microfluidic devices.

To improve mixing between two co-flowing fluids the diffusion distance has to be reduced. This can be achieved by introducing additional flows, time-variations in the overall flow or increasing interfacial area between the flows (Ottino \& Wiggins, 2004). Some example devices based on these strategies include, the serpentine lamination mixer (SLM) which reduces the diffusion distance through increasing interfacial area by taking two parallel flowing fluids, splitting them into multiple smaller segments and then laminating these segments on top of one other (D. S. Kim, Lee, Kwon, \& Ahn, 2004).

Another mixer based on the Coanda effect introduces additional flows by splitting off a part of the flowing fluid. The split part is then reintroduced into the bulk flow further downstream. The split flow is arranged to meet the bulk flow at an angle, thus providing additional transverse flows (C.-C. Hong, Choi, \& Ahn, 2004). 
The final example, a staggered herringbone mixer (SHM) creates time-varying flow by using a series of asymmetric grooves patterned in a channel. The effect of the grooves (see Figure 2) is that two co-flowing fluids are stirred axially as they progress longitudinally through the channel (Stroock et al., 2002). This last strategy to mixing is particularly effective as diffusion distances are reduced exponentially with time. This exponential decrease in diffusion distance is known as 'chaotic mixing' and can also be observed in water droplets flowing in oil (Bringer, Gerdts, Song, Tice, \& Ismagilov, 2004).

The mixer examples described so far are all passive. Another type of mixer, the rotary mixer, uses microvalves to enable active mixing of solutions (Chou, Unger, \& Quake, 2001). In the operation of a rotary mixer solutions are confined to a circular channel. The circular channel contains three valves that are activated in a peristaltic pumping sequence during mixing. The pumping sequence drives the confined solutions around the circular channel causing their mixing. Mixing by this approach is not as rapid as chaotic mixing, but this approach does allow for mixing of discrete segments of fluid whereas the other require continuous flows. Next we look at the automation of microfluidic devices and in particular valve designs that enable functions such as the rotary mixer to be fabricated.

Automation with Valves

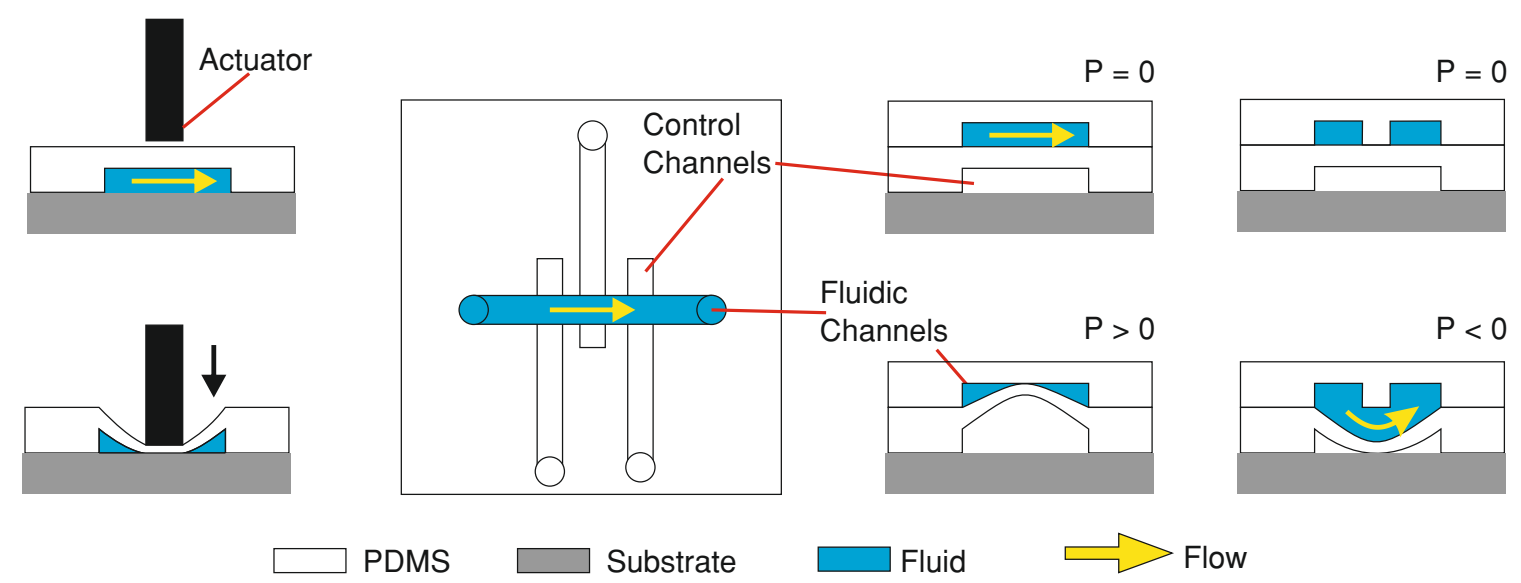

Figure 3. : Microvalves. In (a) viewed from a cross-section, an actuator applies a force directly on to a channel causing it to deform and stop flow. In (b) an in-line valve configuration is depicted from above. In (c) a cross-sections of the in-line valve are shown demonstrating operation. With no pressure applied to the bottom control layer, fluid flow remains unimpeded. On increasing pressure in the control layer the membrane is deflected upwards stopping flow. Similarly in (d) cross-sections of a normally-closed membrane valve are shown. Without pressure, fluid flow remains impeded. On applying vacuum the membrane is deflected away and the fluid is capable of being flowed.

Microvalves have been key to the automation of microfludic devices. Numerous different microvalve designs have been reported, some passive whilst others are actively controlled. Here we focus on a particular type of active valve that is created by sandwiching a flexible 
membrane between two layers containing microchannels (see Figure 3). For a more complete review of microvalves see (Oh \& Ahn, 2006). The type of microvalve we are interested in is sometimes referred to as a membrane or in-line valve (Oh \& Ahn, 2006). This type of microvalve has allowed for microfluidic devices of previously unachievable levels of automation. The reason behind their success has been the ability to pack the microvalves in close proximity, for example within $50 \mu \mathrm{m}$ of each other as reported in (Thorsen, Maerkl, \& Quake, 2002). This packing density has been achieved by moving the relatively bulky valve driving component, the pressure or vacuum source, away from the microvalve. Instead, 'control' microchannels are created that link the on-chip microvalve to the driving component at the periphery of the chip. By contrast other active microvalves have the external work source directly acting on a fluidic channel (Weibel et al., 2005).

The in-line valve design introduced by Quake's group (Unger, Chou, Thorsen, Scherer, \& Quake, 2000) has been shown to enable construction of on-chip components such as peristaltic pumps and rotary mixers (Chou et al., 2001). Additionally this in-line valve has been shown capable of being multiplexed so that large numbers of fluidic channels are controlled by a small number of control channels; one example demonstrates 20 control channels addressing 1024 fluidic channels (Thorsen et al., 2002). Such microfluidic largescale integrated (mLSI) schemes in combination with pumping and mixing components have allowed for complex devices to be created capable of performing parallel operations on a single chip (J. W. Hong, Studer, Hang, Anderson, \& Quake, 2004; Jambovane, Duin, Kim, \& Hong, 2009).

The device complexity that can be achieved and the rapid prototyping techniques available to the creation of in-line valves makes them attractive for automated device design. Particularly so when fluids are to be handled as segments or chemical vessels. But as we shall see next, there are some issues to consider before employing in-line valves as a means to creating and handling chemical vessels.

\section{Creating Chemical Vessels}

So far we have considered microfluidic systems that manipulate single-phase aqueous solutions. Most of the example microfluidic systems in our discussion operate with continuous flow. Where individual samples of solution are required, valves can be employed as in the case of the rotary mixer. However using valves to obtain a segment or vessel of fluid inevitably leads to more complex designs and fluid wastage.

For example consider a channel with valves at its entrance and exit separated by a distance $L$. On actuation of the valves a fluid segment will be obtained equal to the distance between the valves, i.e. of length $L$. To obtain the section of fluid requires the complete filling the channel. This is achieved by flowing the fluid beyond the exit valve. When the valves are actuated, any excess fluid beyond the exit valve is then wasted. Now if multiple fluids are to be segmented as in the case of (J. W. Hong et al., 2004), the device design is complicated as inlets and outlets are required for each segmentation channel. Normally junctions containing multiple valves are used.

There are other issues that require consideration when dealing with single-phase flows. For example when using the same channels for different fluids there is a risk of crosscontamination of samples. Additionally, sufficiently long segments of channel can lead to 
measurement errors due to Taylor dispersion. Taylor dispersion is a phenomena associated with pressure-driven flow. If we observe a uniform plug of molecules in an aqueous pressure-driven flow, the plug is subjected to longitudinal stretching along the direction of flow. The stretching of the plug when viewed along the length of the channel has a characteristic parabolic shape. Concentration gradients are present at the front and rear of the parabola. Diffusion acts to smear the gradients causing a wider more dilute plug to emerge. The process is repeated with the original uniform plug evolving as a widening plug with a concentration akin to a Gaussian distribution.

Taylor dispersion occurs only when molecules have completely mixed across the width of a microchannel. This corresponds to a time scale $t \gg \frac{w^{2}}{D}$ or a downstream distance of $L \gg P e \cdot w$ (Squires \& Quake, 2005) - from the previous examples, $620 \mathrm{~s}$ and $6.2 \mathrm{~cm}$ respectively. In theory Taylor dispersion can be accommodated by employing sufficiently slow flows and/or thin channels where $P e \leq 1$. In this case a plug of molecules would evolve as though the flow had a uniform profile. However mixing would take too long and therefore renders these options unrealistic.

All of these issues can be addressed by introducing an additional phase to a microfluidic flow. For example a two-phase flow can be created by using oil as a carrier fluid into which aqueous droplets acting as self-contained chemical reaction vessels can be injected. The same concepts of laminar flow, fluidic resistance and mixing described previously still apply to twophase flows. There are however more concepts to consider when dealing with droplets which are beyond the scope of this paper (for further information see (Song, Chen, \& Ismagilov, 2006)).

As for the in-line valve designs we described earlier, these can and have been implemented in microfluidic droplet devices. In-line valves have allowed for the control over injected droplet size (Zeng, Li, Su, Qin, \& Lin, 2009) as well as the control of mixing (or fusion) of two or more droplets together (B.-C. Lin \& Su, 2008). Therefore the concepts of valves described earlier are relevant to droplet devices. Next we discuss the fabrication techniques and materials typically employed in the fabrication of single-phase, droplet and in-line valved devices.

\section{Microfluidic Device Fabrication}

Microchannels can be patterned in substrate materials that include glass (C.-H. Lin, Lee, Lin, \& Chang, 2001), silicon (Harris et al., 2003) and polymeric materials such as elastomers and thermoplastics (Becker \& Gärtner, 2008). Of the substrate materials used, the silicone elastomer polydimethylsiloxane (or PDMS) is arguably the most widely employed material in the fabrication of microfluidic devices. Therefore we choose our attention on the fabrication of microfluidic devices in PDMS.

Reasons for the popularity of PDMS in microfluidics may include, the availability of relatively quick and easy prototyping methods (Duffy, McDonald, Schueller, \& Whitesides, 1998), the ability to support the creation of high density valved devices (Thorsen et al., 2002), little optical absorbance in the range of wavelengths between $230 \mathrm{~nm}$ and $700 \mathrm{~nm}$ (Fujii, 2002), biocompatibility (Folch \& Toner, 1998), modifiable surface wettability (Bhattacharya, Datta, Berg, \& Gangopadhyay, 2005) and reasonable solvent compatibility (Lee, Park, \& Whitesides, 2003). 
i.

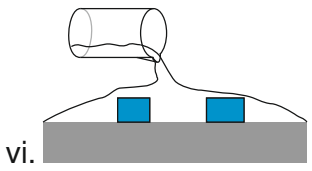

ii.
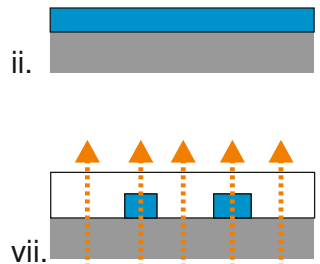

iii.

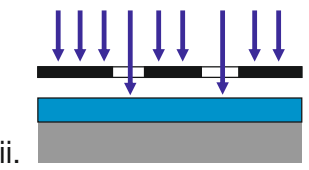

viii

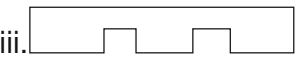

$\square$ PDMS iv.

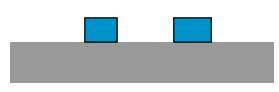

ix.

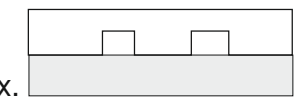

Glass

Figure 4. : Summary of the fabrication processes of replication masters and PDMS devices. In (i.-iv.) a substrate is coated with a photoresist. The photoresist is selectively exposed to UV light through a photomask. The resist that remains after developing dictates the pattern to be replicated. In (vi.-ix.) prepared PDMS is poured on to a replication master. The PDMS coated replication master is then baked allowing the PDMS to cure. The cured PDMS is then released from the replication master. Afterwards the cured PDMS is then bonded, in this case to glass, sealing the microchannels and constituting a microfluidic device.

Prototyping in PDMS involves three major steps, replication master fabrication, patterning and bonding. The first step of replication master fabrication requires the desired pattern to be obtained in PDMS to be created on a substrate. As this step determines the fidelity of the resulting microstructures in PDMS, it tends to be the most time consuming and expensive step. Many of the techniques used for replication master fabrication have been borrowed from the integrated circuit industry. Similarly replication master fabrication is often performed in specialist cleanroom environments using sophisticated equipment (e.g. Standford's Microfluidic Foundry and Southampton's Nanofabrication Centre).

Photolithography is perhaps the mostly widely used method to replication master creation. Following Figure ??, in master fabrication, typically liquid photoresists are deposited on to a silicon wafer by spin-coating. Both positive (e.g. AZ series, MicroChemicals GmbH, Germany for rounded channels, see (Unger et al., 2000)) and negative (e.g. SU-8 series, MicroChem Corp., USA) tone photoresists are used. A photomask is then used to selectively block ultraviolet light that is brought incident to the deposited photoresist. This is often performed within a mask-aligner containing a collimated UV light source. A developing step is then performed after exposure of the photoresist to UV light. During developing excess resist material is etched away yielding a patterned silicon wafer. Normally the patterned wafer is then subjected to a surface treatment (silanization) to render it non-stick to PDMS, which aids its removal after curing.

When the replication master is complete, PDMS is prepared and poured on to the replication master. The PDMS is then cured at an elevated temperature within an oven or on a hotplate. After curing the PDMS is released from the replication master. Fluid access holes are then made in the cured PDMS using a coring tool, drill or punching with a needle. The PDMS is then ready to be bonded.

PDMS can bond reversibly by simply bringing it into conformal contact with a sub- 
strate such as glass, silicon or another piece of PDMS. For applications that involve higher operating pressures irreversible bonding of PDMS is generally required. Irreversible bonding techniques in general either involve surface treatments (Haubert, Drier, \& Beebe, 2006), adhesives in the form of uncured PDMS or curing agent (Satyanarayana, Karnik, \& Majumdar, 2005; Samel, Chowdhury, \& Stemme, 2007) or variation of the PDMS processing parameters, for example temperature or ratio of curing agent (Unger et al., 2000; Go \& Shoji, 2004).

A particularly popular and effective method of bonding involves treating PDMS with an $\mathrm{O}_{2}$ plasma generated by a plasma cleaning machine. Exposure to plasma forms reactive silanol $(-\mathrm{OH})$ groups on the surface of the PDMS and also renders the PDMS hydrophilic from its natural hydrophobic state. By bringing two oxidised PDMS pieces into contact, it is believed that covalent siloxane ( $\mathrm{Si}-\mathrm{O}-\mathrm{Si}$ ) bonds are formed through a condensation reaction giving rise to the irreversible seal (Duffy et al., 1998). Plasma treatment can also be used to irreversibly bond PDMS to glass. Both the PDMS and glass require exposure to the $\mathrm{O}_{2}$ plasma.

There is an issue with bonding by plasma treatment when multiple device layers are to be aligned and bonded. Oxidised PDMS layers when brought into contact with one another or glass results in almost immediate bonding. There is normally only one chance to align layers correctly before bringing them into contact. Thus alignment is tricky. Solutions have be found in using alignment equipment (for example (Moraes, Sun, \& Simmons, 2009)), but there is a limited lifespan (normally several minutes) to the oxidised state of the PDMS layers after which bonding is no longer possible. An alternate method uses methanol which temporarily inhibits bonding between oxidised layers (Jo, Lerberghe, Motsegood, \& Beebe, 2000). However using methanol can result in the swelling of PDMS (Lee et al., 2003).

\section{Characterising Enzymes for Information Processing}

Much of the information processing within biological systems can be attributed to the interactions of protein macromolecules (Bray, 1995). The proteins known as enzymes in particular can be viewed as spatial computers with physical structures sensitive to the chemical environment in which they reside. Characterising the response behaviour of single and networks of enzymes to chemical inputs could lead to new modes of information processing being discovered (Zauner \& Conrad, 2001). Performing the characterisation is by no means simple. Enzyme response behaviour is in itself nonlinear. Thus it is not possible to reduce the number of enzyme characterisation experiments to be performed by for example, factorial design. Or at least not without the risk of missing potentially important information. Therefore detailed enzyme characterisation is required. However detailed investigation is in turn limited by the availability of chemical resources, the complexity of experimentation protocols and the ability to analyse large volumes of data. A means to addressing these limits and enabling detailed investigation of enzyme behaviour may be found in autonomous experimentation.

\section{Autonomous Experimentation}

In autonomous experimentation, machine learning algorithms are coupled in a closedloop manner to an automated experimentation platform. Following Figure 5, the basic concept is that the machine learning algorithm formulates hypotheses, tests them through 
Machine Learning Algorithms

Automated Experimentation

Hardware

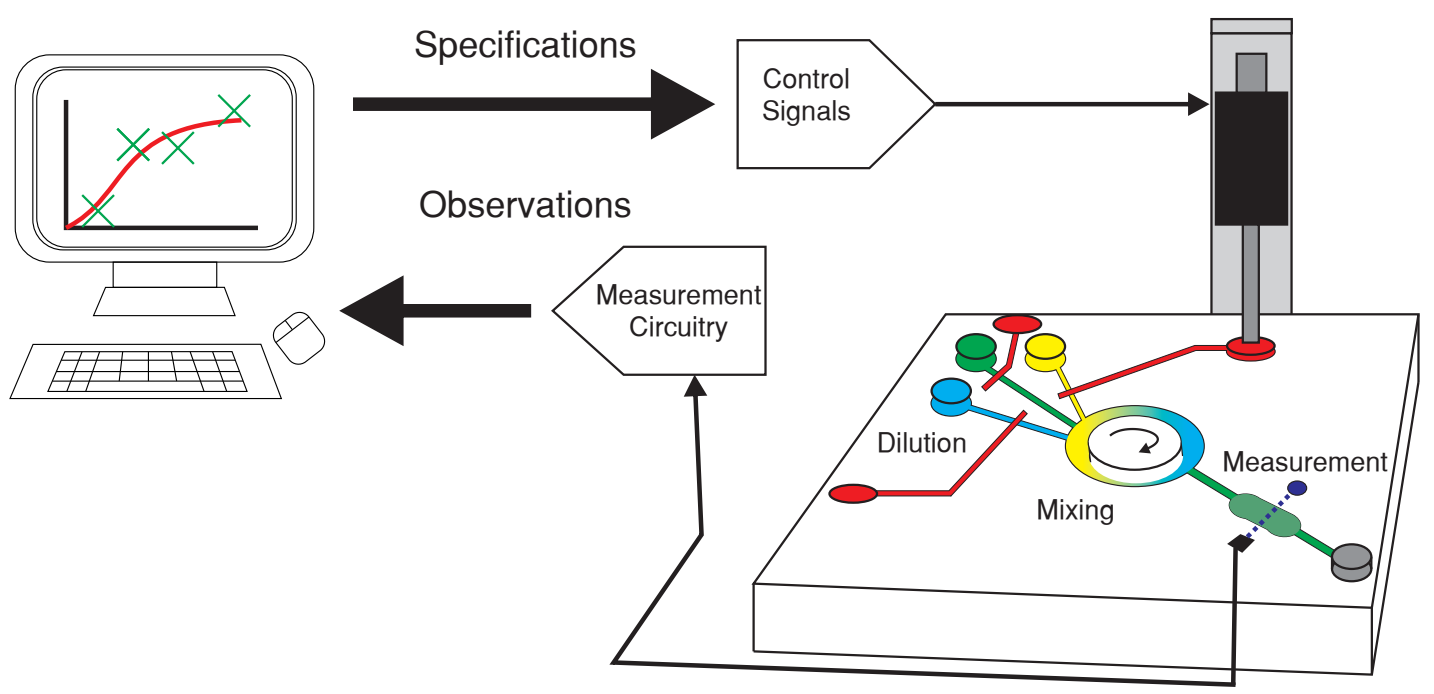

Figure 5. : The conceptual autonomous experimentation system with microfluidic-based hardware.

the automated experimentation platform, analyses measurements and then reformulates hypotheses for further testing.

The algorithms we use for autonomous experimentation are described elsewhere (Lovell, Jones, Gunn, \& Zauner, 2010). Here we focus on the development of the experimentation platform and begin by considering some of the basic requirements to perform enzymatic assays.

\section{Enzyme Assay Requirements}

The study reported in (Zauner \& Conrad, 2001) explored the response of the enzyme malate dehydrogenase $(\mathrm{MDH})$ to chemical inputs in the form of the metal ions $\mathrm{Ca}^{2+}$ and $\mathrm{Mg}^{2+}$. Normally the enzyme malate dehydrogenase (MDH) catalyses the oxidation of malate to oxalacetate by reducing nicotinamide adenine dinucleotide (NAD+) to NADH (Englard \& Siegel, 1969):

$$
\mathrm{L}-\text { malate }+\mathrm{NAD}^{+} \stackrel{M D H}{\longleftrightarrow} \text { oxalacetate }+\mathrm{NADH}+\mathrm{H}^{+}
$$

where NADH differs significantly in its absorbance of ultraviolet light at $340 \mathrm{~nm}$ when compared to $\mathrm{NAD}^{+}$, which provides an optical measure of the catalytic activity or enzyme response. In the study, the introduction of dilutions of the metal ions altered the enzyme response in a way that was analysed and interpreted as Boolean logic. The basic requirements for the enzymatic assays in the study were identified as means to dilute the inputs, mix all chemicals and measure the catalytic activity of the enzyme. The experimentation platform 
therefore has to fulfil these basic requirements, whilst also addressing the issues of resource limitation and experimentation protocol complexity as mentioned previously. By adopting microfluidic technology it should be possible for all of these issues to be addressed.

\section{Microfluidics for Autonomous Experimentation}

In our adoption of microfluidic technology in autonomous experimentation we investigated low-cost methods for fabrication and interfacing to insure that the approach can be scale to many channels of different signaling chemicals. We consider the outcomes of our investigations next.

\section{Fabrication}

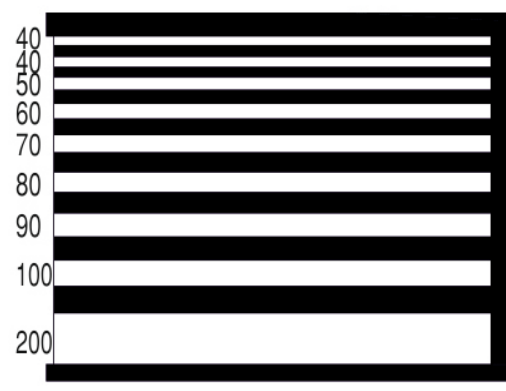

(a)

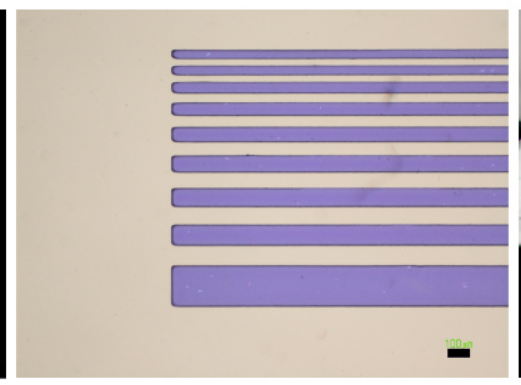

(b)

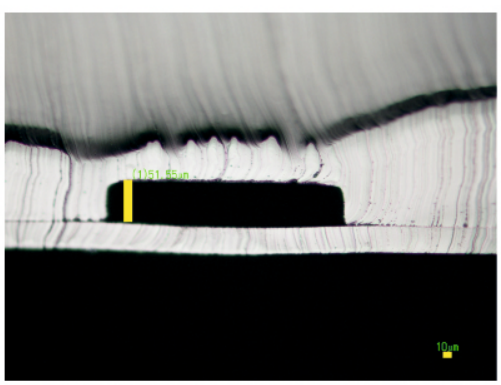

(c)

Figure 6. : Results from characterising dry film resists. A section of the photomask used to characterise the dry film resists is shown in (a). The white lines represent clear parts of the mask. The numbers to the left of the lines indicate their width in microns. In (b) we see the resulting pattern replicated in a dry film resist that was laminated onto a glass substrate. The horizontal scale bar represents $100 \mu \mathrm{m}$. In (c) is a cross-section of PDMS bonded to a thin PDMS film. The channel was patterned using a dry film replication master. The vertical scale bar represents $\approx 50 \mu \mathrm{m}$. It can be seen from the cross-section that the channel sidewalls are reasonably square despite the replication master being created using an uncollimated light source.

As previously described, replication master fabrication can impose major economic constraints due to the requirements of sophisticated equipment and facilities. Searching for alternatives we have found that using dry film resists (DFRs) in place of liquid resists could provide up to a ten-fold saving in material costs alone. The equipment required for processing DFRs does not need to be sophisticated. Normally dry film resists are deposited on to a substrate through lamination where an office hot roll laminator will suffice (Vulto et al., 2005).

Dry film resists have been reported as being used in the fabrication of microfluidic devices. This has been achieved by either patterning channels in the resist itself (Vulto et al., 2005) or by using the resist as part of a replication master. DFR-based replication masters have been used for hot embossing plastics such as PMMA (Sandison \& Morgan, 2005) and patterning PDMS (Stephan et al., 2007). From these examples dry film resists 
were reported to be capable of yielding structures of aspect ratios (height to width) as high as 2.8:1 and as low as 1.2:1 (Vulto et al., 2005). The smallest features possible with DFRs reported in (Vulto et al., 2005) were $20 \mu \mathrm{m}$ with $40 \mu \mathrm{m}$ spacing between them, whilst in (Stephan et al., 2007) $30 \mu \mathrm{m}$ features with $30 \mu \mathrm{m}$ spacing were possible with a collimated light source (mask aligner). These dimensions easily support microfluidic in-line valves, which in themselves can be as large as a few hundred microns.

We have found dry film resists to be an effective alternative to liquid resists, particularly when laminated on glass microscope slides. We have found that we can produce features down to as small as $40 \mu \mathrm{m}$ in width (see Figure ??) without a collimated light source (Stephan et al., 2007) could get no smaller than $200 \mu \mathrm{m}$ without a collimated light source. Recognising the potential for our DFR protocols to be useful for others we have included them in the Appendix.

We have also found an alternate means to aligning plasma treated PDMS. Having tried the methanol lubrication approach described by (Jo et al., 2000) we found limited success in bonding. We have since discovered that substituting the methanol for water and by following a series of water purging steps (see Appendix C), alignment and good irreversible bonding is achievable.

On-Chip Valve Interfacing and Prototype System

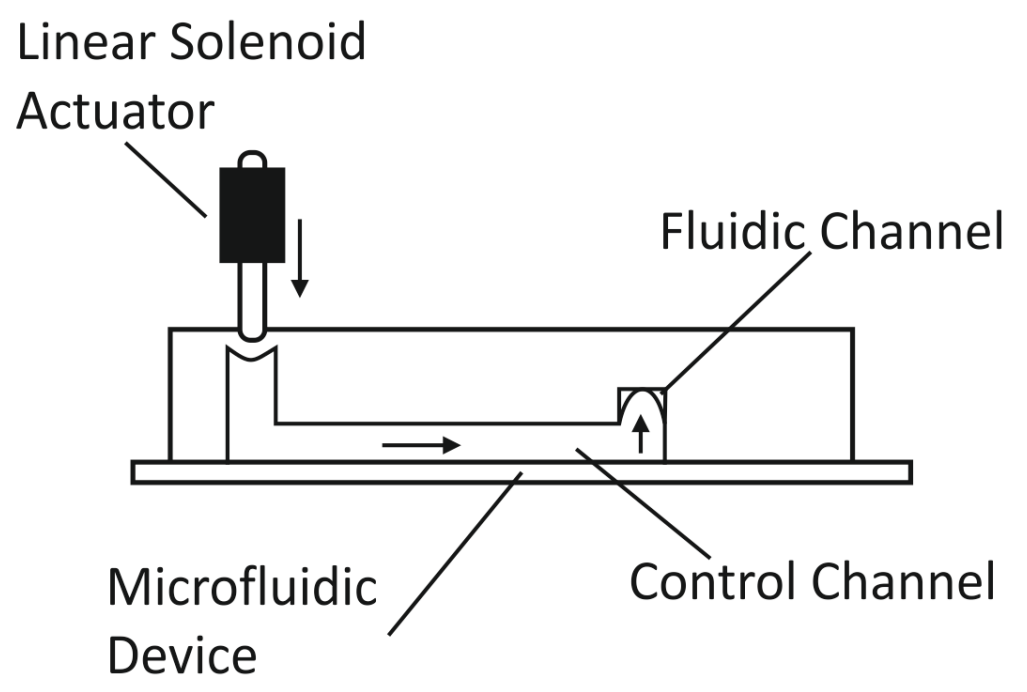

Figure 7. : Electrohydraulic interface. A linear motion solenoid applies pressure on to a sealed hydraulic control channel. The force is transmitted by the hydraulic fluid and deflects the membrane closing the valve.

In characterising enzymes we have established the functions dilution, mixing and measurement are required. For increased computative complexity, multiple chemical signals are to be used and consequently multiple microfluidic channels and associated pumps are required. Thus a low cost implementation is desirable. Current approaches to interfacing 
and controlling on-chip valves predominantly use moderately expensive pneumatic actuators (Urbanski, Thies, Rhodes, Amarasinghe, \& Thorsen, 2006) but in some instances Braille displays are used (Gu, Zhu, Futai, Cho, \& Takayama, 2004; Futai, Gu, Song, \& Takayama, 2006; Gu, Chen, Tung, Meiners, \& Takayama, 2007). While the cost per actuator of the Braille display approach is lower than that of the pneumatic, it lacks the freedom of pneumatic actuation in allowing for arbitrary placement of the actuators. The approach presented here addressed both issues.

We have developed a new type of electrohydraulic interface enabling low-cost computer control over valved microfluidic chips. The interface scheme is illustrated in Figure 7 and uses low-cost ( $<£ 9$ each) linear action solenoids operating on a sealed microfluidic control channel. The interface scheme has been tested on a prototype valved microfluidic device and the results are shown in Figure 8.

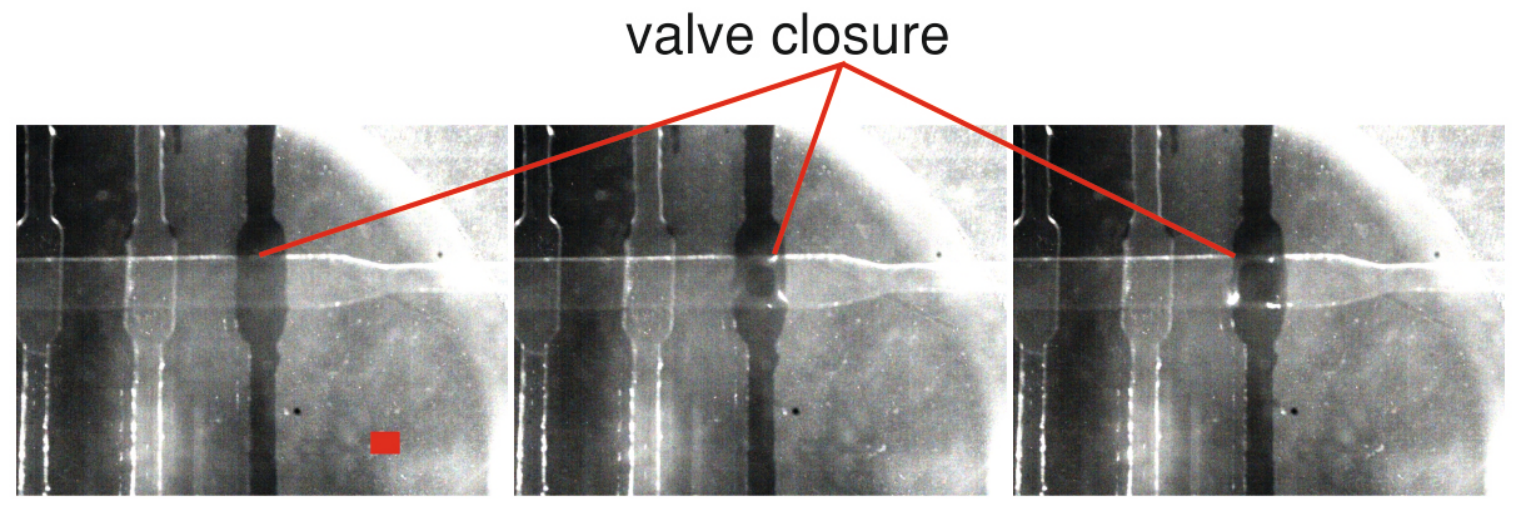

Figure 8. : Photographic sequence showing on-chip valve actuation with force applied by a linear motion solenoid. The control line is seen in black intersecting the transparent fluidic channel. The voltage applied to the solenoid was varied between $0 \mathrm{~V}$ and $6 \mathrm{~V}$. In (a), at $0 \mathrm{~V}$ the valve was completely open. From (b) to (c) the valve progressively closed with increasing voltage recognisable by the dark area and distortion around it appearing in the centre of the valve. The scale bar represents $400 \mu \mathrm{m}$.

To give the solenoids flexibility of placement, they are mounted on handmade L-shaped aluminium brackets with height adjustment slots. Neodymium magnets are glued to the base of the brackets allowing for solenoid positioning around a microfluidic chip placed on a sheet of galvanised steel (see Figure 9c). The magnets allow for the position of the solenoids to be fixed and moved as necessary.

We have also been developing the measurement function of the experimentation platform. To provide measurements a photometer has been developed to monitor optical transmission through a detection path on the microfluidic chip. The photometer design uses an ultraviolet light emitting diode and an ultraviolet sensitive photodiode through which a modulated optical signal is transmitted and received. The optical signal is generated and filtered by a low-cost lock-in amplifier implemented in a digital signal processor. The lock-in amplifier communicates optical measurements to a personal computer where a low-level software interface captures the optical measurements as well as providing control over the valves and pumps. A simple custom scripting language was developed for the low-level software 
interface allowing for a set of valves to be actuated as a pump and measurements to be taken, all by single commands. In Figure 9b the entire prototype experimentation system is shown.

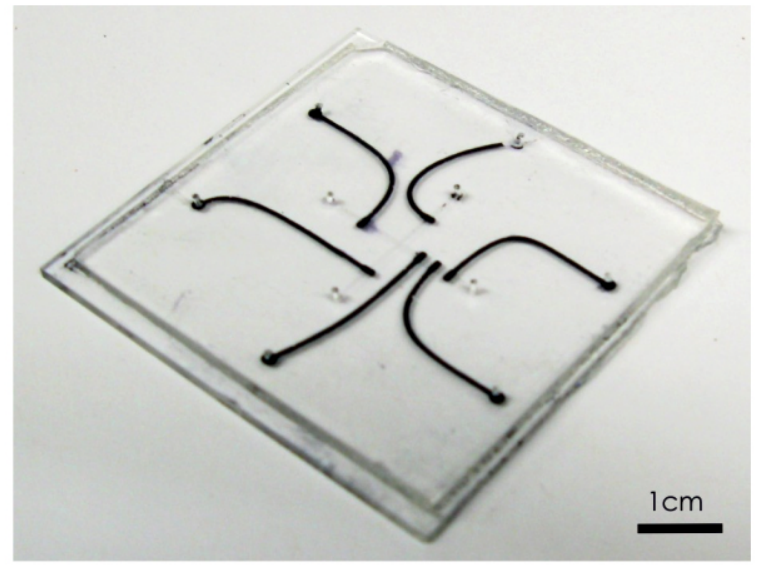

(a)

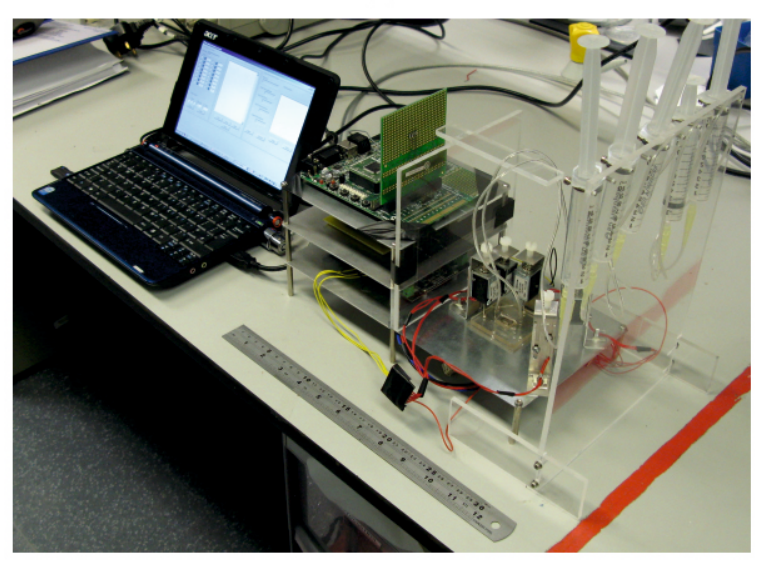

(b)

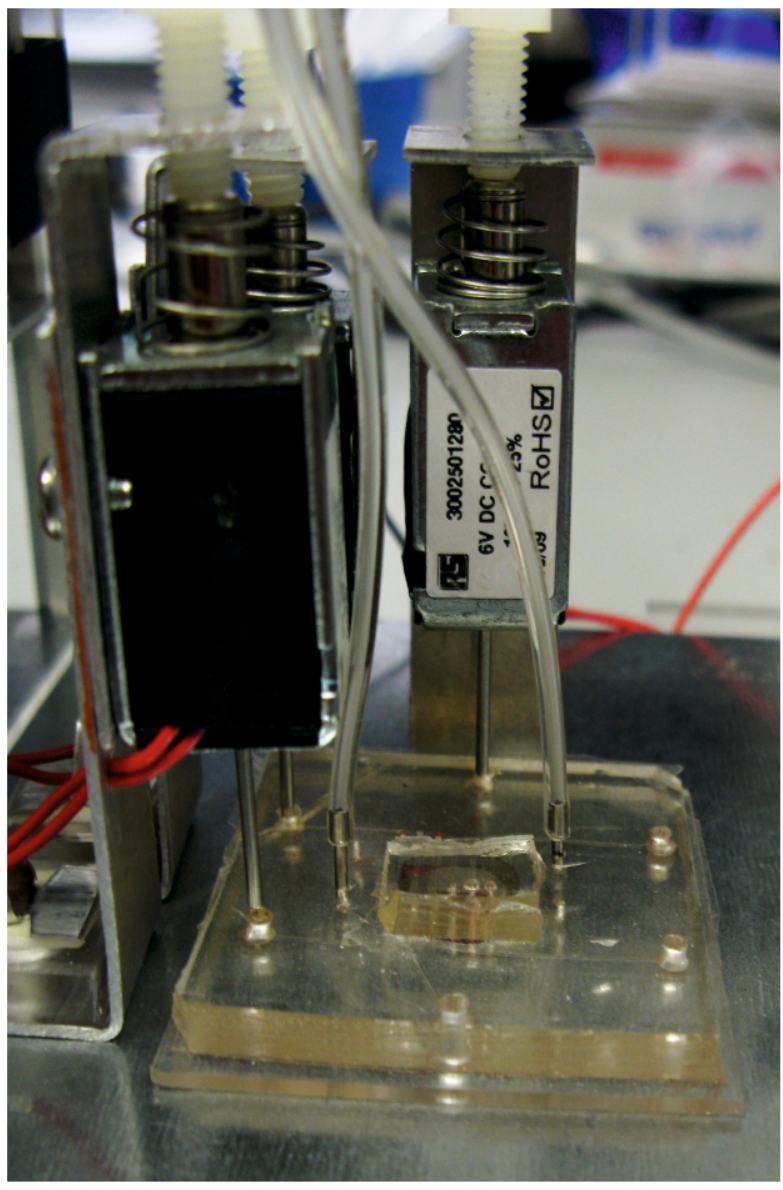

(c)

Figure 9. : Aspects of the prototype automated experimentation system. In (a) is an early prototype valved device with control channels filled with black ink for visibility. Shown in (b) is the overall automated experimentation system hardware and control software. In (c) we see a close-up of the solenoid actuators positioned around a prototype microfluidic device.

\section{Concluding Discussion}

Biomolecules offer a viable substrate for information processing as evidenced in nature. Macromolecules such as enzymes are rich in functionality and interactions. At present it is not possible to design enzymes for information processing. It is, however, possible to employ natural enzymes. To fully exploit the possibility offered by natural enzymes, it is necessary to elicit their response to chemical signals that could see an application in enzymatic computers. The range of potential chemical signals and their concentrations span a very large space of possibilities. The task at hand, then is to use the available material, time, and equipment 
resources to discover as much useful functionality in existing biomacromolecules as possible. To answer this challenge we have developed an autonomous experimentation system that combines machine learning with computer controlled microfluidics. In both areas we found that the existing methods did not meet our needs. We developed learning techniques suitable for small numbers of noisy samples (Lovell et al., 2010) and developed the microfluidic technology described here to make studies with many signaling molecules affordable.

The design of our experimentation platform focused on low-cost pumps and valves to maximise the number of chemical signals that can be used. The technology needed to fabricate the microfluidic chips with the proposed method is not very sophisticated and should allow for easy adoption by other groups. With the combination of low-cost fabrication techniques and a low-cost computer controlled actuation scheme, enzymatic computing studies should become feasible that were previously unaffordable.

\section{References}

Baron, R., Lioubashevski, O., Katz, E., Niazov, T., \& Willner, I. (2006a). Elementary arithmetic operations by enzymes: A model for metabolic pathway based computing. Angew. Chem. Int. Ed., 45, 1572-1576.

Baron, R., Lioubashevski, O., Katz, E., Niazov, T., \& Willner, I. (2006b). Logic gates and elementary computing by enzymes. J. Phys. Chem. A, 110, 8548-8553.

Baron, R., Lioubashevski, O., Katz, E., Niazov, T., \& Willner, I. (2006c). Two coupled enzymes perform in parallel the 'and' and 'inhiband' logic gate operations:. Organic and Biomolecular Chemistry, 4, 989-991.

Becker, H., \& Gärtner, C. (2008). Polymer microfabrication technologies for microfluidic systems. Analytical and Bioanalytical Chemistry, 390, 89-111.

Beebe, D. J., Mensing, G. A., \& Walker, G. M. (2002). Physics and applications of microfluidics in biology. Annual Review of Biomedical Engineering, 4, 261-286.

Bhattacharya, S., Datta, A., Berg, J. M., \& Gangopadhyay, S. (2005, June). Studies on surface wettability of poly(dimethyl)siloxane (PDMS) and glass under oxygen-plasma treatment and correlation with bond strength. Journal of Microelectromechanical Systems, 14(3), 590-597.

Bray, D. (1995). Protein molecules as computational elements in living cells. Nature, 376, 307-312.

Bringer, M. R., Gerdts, C. J., Song, H., Tice, J. D., \& Ismagilov, R. F. (2004). Microfluidic systems for chemical kinetics that rely on chaotic mixing in droplets. Philisophical Transactions of the Royal Society of London A, 362, 1087-1104.

Chou, H.-P., Unger, M. A., \& Quake, S. R. (2001). A microfabricated rotary pump. Biomedical Microdevices, 3(4), 323-330.

Duffy, D. C., McDonald, J. C., Schueller, O. J. A., \& Whitesides, G. M. (1998). Rapid prototyping of microfluidic systems in poly(dimethylsiloxane). Analytical Chemistry, 70 (23), 4974-4984.

Englard, S., \& Siegel, L. (1969). Mitochondrial l-malate dehydrogenase of beef heart. In J. M. Lowenstein (Ed.), Citric acid cycle (Vol. XIII). Academic Press, New York.

Folch, A., \& Toner, M. (1998). Cellular micropatterns on biocompatible materials. Biotechnol. Prog., 14, 388-392.

Fujii, T. (2002). PDMS-based microfluidic devices for biomedical applications. Microelectronic Engineering, 61-62, 907-914.

Futai, N., Gu, W., Song, J. W., \& Takayama, S. (2006). Handheld recirculation system and customized media for microfluidic cell culture. Lab on a Chip, 6, 149-154. 
Go, J. S., \& Shoji, S. (2004). A disposable, dead volume-free and leak-free in-place PDMS microvalve. Sensors and Actuators A, 114, 438-444.

Gu, W., Chen, H., Tung, Y.-C., Meiners, J.-C., \& Takayama, S. (2007). Multiplexed hydraulic valve actuation using ioninc filled soft channels and braille displays. Applied Physics Letters, 90, 033505-1-033505-3.

Gu, W., Zhu, X., Futai, N., Cho, B. S., \& Takayama, S. (2004). Computerized microfluidic cell culture using elastomeric channels and braille displays. PNAS, 101, 15861-15866.

Harris, N. R., Hill, M., Beeby, S., Shen, Y., White, N. M., Hawkes, J. J., et al. (2003). A silicon microfluidic ultasonic separator. Sensors and Actuators B, 95, 425-434.

Haubert, K., Drier, T., \& Beebe, D. (2006). PDMS bonding by means of a portable, low-cost corona system. Lab on a Chip, 6, 1548-1549.

Hong, C.-C., Choi, J.-W., \& Ahn, C. H. (2004). A novel in-plane passive microfluidic mixer with modified Tesla structures. Lab on a Chip, 4, 109-113.

Hong, J. W., Studer, V., Hang, G., Anderson, W. F., \& Quake, S. R. (2004, April). A nanoliter-scale nucleic acid processor with parallel architecture. Nature Biotechnology, 22(4), 435-439.

Jambovane, S., Duin, E. C., Kim, S.-K., \& Hong, J. W. (2009). Determination of kinetic parameters, $\mathrm{k}_{m}$ and $\mathrm{k}_{\text {cat }}$, with a single experiment on a chip. Analytical Chemistry, 81(9), 3239-3245.

Jeon, N. L., Dertinger, S. K. W., Chiu, D. T., Choi, I. S., Stroock, A. D., \& Whitesides, G. M. (2000). Generation of solution and surface gradients using microfluidic systems. Langmuir, $16,8311-8316$.

Jo, B.-H., Lerberghe, L. M. V., Motsegood, K. M., \& Beebe, D. J. (2000, March). Three-dimensional micro-channel fabrication in polydimethylsiloxane (PDMS) elastomer. Journal of Microelectromechanical Systems, 9(1), 79-81.

Kim, C., Lee, K., Kim, J. H., Shin, K. S., Lee, K.-J., Kim, T. S., et al. (2008). A serial dilution microfluidic device using a ladder network generating logarithmic or linear concentrations. Lab on a Chip, 8, 473-479.

Kim, D. S., Lee, S. H., Kwon, T. H., \& Ahn, C. H. (2004). A serpentine laminating micromixer combining splitting/recombination and advection. Lab on a Chip, 5, 739-747.

Kim, L., Vahey, M. D., Lee, H.-Y., \& Voldman, J. (2006). Microfluidic arrays for logarithmically perfused embryonic stem cell culture. Lab on a Chip, 6, 394-406.

Kovacs, G. T. (1998). Micromachined transducers sourcebook. McGraw-Hill Science.

Lee, J. N., Park, C., \& Whitesides, G. M. (2003). Solvent compatibility of poly(dimethylsiloxane)based microfluidic devices. Analytical Chemistry, 75 (23), 6544-6554.

Lin, B.-C., \& Su, Y.-C. (2008). On-demand liquid-in-liquid droplet metering and fusion utilizing pneumatically actuated membrane valves. Journal of Micromechanics and Microengineering, $18,1-10$.

Lin, C.-H., Lee, G.-B., Lin, Y.-H., \& Chang, G.-L. (2001). A fast prototyping process for fabrication of microfluidic systems on soda-lime glass. J. Micromech. Microeng., 11, 726-732.

Lovell, C., Jones, G., Gunn, S., \& Zauner, K.-P. (2010, November). An artificial experimenter for enzymatic response characterisation. 13th International Conference on Discovery Science, $42-56$.

Moraes, C., Sun, Y., \& Simmons, C. A. (2009). Solving the shrinkage-induced PDMS alignment registration issue in multilayer soft lithography. Journal of Micromechanics and Microengineering , 19, 1-6.

Niazov, T., Baron, R., Katz, E., Lioubashevski, O., \& Willner, I. (2006). Concatenated logic gates using four coupled biocatalysts operating in series. Proceedings of the National Academy of Sciences, 103(46), 17160-17163. 
Oh, K. W., \& Ahn, C. H. (2006). A review of microvalves. Journal of Micromechanics and Microengineering, 16, R13-R39.

O'Neill, A., Hoo, J. S., \& Walker, G. (2006). Chips \& tips: Rapid curing of PDMS for microfluidic applications. Lab on a Chip.

Ottino, J. M., \& Wiggins, S. (2004). Introduction: mixing in microfluidics. Philisophical Transactions of the Royal Society of London A, 362, 923-935.

Pita, M., Zhou, J., Manesh, K. M., Halámek, J., Katz, E., \& Wang, J. (2009). Enzyme logic gates for assessing physiological conditions during an injury: Towards digital sensors and actuators. Sensors and Actuators B, 139, 631-636.

Samel, B., Chowdhury, M. K., \& Stemme, G. (2007). The fabrication of microfluidic structures by means of full-wafer adhesive bonding using a poly(dimethysiloxane) catalyst. Journal of Micromechanics and Microengineering, 17, 1710-1714.

Sandison, M. E., \& Morgan, H. (2005). Rapid fabrication of polymer microfluidic systems for the production of artifical lipid bilayers. J. Micromech. Microeng., 15, S139-S149.

Sandison, M. E., Zagnoni, M., Abu-Hantash, M., \& Morgan, H. (2007). Micromachined glass apertures for artificial lipid bilayer formation in a microfluidic system. Journal of Micromechanics and Microengineering, 17, S189-S196.

Satyanarayana, S., Karnik, R. N., \& Majumdar, A. (2005, April). Stamp-and-stick room temperature bonding technique for microdevices. Journal of Microelectromechanical Systems, 14(2), 392-399.

Sivan, S., Tuchman, S., \& Lotan, N. (2003). A biochemical logic gate using an enzyme and its inhibitor. part ii: The logic gate. BioSystems, 70, 21-33.

Song, H., Chen, D. L., \& Ismagilov, R. F. (2006). Reactions in droplets in microfluidic channels. Angewandte Chemie Int. Ed., 45, 7336-7356.

Squires, T. M., \& Quake, S. R. (2005). Microfluidics: fluid physics at the nanoliter scale. Reviews of Modern Physics, 77, 977-1026.

Stephan, K., Pittet, P., Renaud, L., Kleimann, P., Morin, P., Ouaini, N., et al. (2007). Fast prototyping using a dry film photoresist: microfabrication of soft-lithography masters for microfluidic structures. Journal of Micromechanics and Microengineering, 17, N64-N74.

Stroock, A. D., Dertinger, S. K. W., Ajdari, A., Mezić, I., Stone, H. A., \& Whitesides, G. M. (2002, January 25). Chaotic mixer for microchannels. Science, 295, 647-651.

Thorsen, T., Maerkl, S. J., \& Quake, S. R. (2002). Microfluidic large-scale integration. Science, $298,580-584$.

Unger, M. A., Chou, H.-P., Thorsen, T., Scherer, A., \& Quake, S. R. (2000, April 7th). Monolithic microfabricated valves and pumps by multilayer soft lithography. Science, 288, 113-116.

Urbanski, J. P., Thies, W., Rhodes, C., Amarasinghe, S., \& Thorsen, T. (2006). Digital microfluidics using soft lithography. Lab on a Chip, 6, 96-104.

Vulto, P., Glade, N., Altomare, L., Bablet, J., Tin, L. D., Medoro, G., et al. (2005). Microfluidic channel fabrication in dry film resist for production and prototyping of hybrid chips. Lab on a Chip, 5, 158-162.

Walker, G. M., Monteiro-Riviere, N., Rouse, J., \& O’Neill, A. T. (2007). A linear dilution microfluidic device for cytotoxicity assays. Lab on a Chip, 7, 226-232.

Weibel, D. B., Kruithof, M., Potenta, S., Sia, S. K., Lee, A., \& Whitesides, G. M. (2005, August 1st). Torque-actuated valves for microfluidics. Analytical Chemistry, 7r7(15), 4726-4733.

Zauner, K.-P., \& Conrad, M. (2001). Enzymatic computing. Biotechnol. Prog., 17, 553-559.

Zeng, S., Li, B., Su, X., Qin, J., \& Lin, B. (2009). Microvalve-actuated precise control of individual droplets in microfluidic devices. Lab on a Chip, 9, 1340-1343. 
Zhang, W.-Y., Ferguson, G. S., \& Tatic-Lucic, S. (2004, January 25-29). Elastomer-supported cold welding for room temperature wafer-level bonding. In Technical digest of 17th IEEE international conference on micro electro mechanical systems (mems 2004) (pp. 741-744). Maastricht, The Netherlands.

Zhou, J., Tam, T. K., Pita, M., Ornatska, M., Minko, S., \& Katz, E. (2009). Bioelectrocatalytic system coupled with enzyme-based biocomputing ensembles performing boolean logic operations: approaching "smart" physiologically controlled biointerfaces. Applied Materials and Interfaces, 1(1), 144-149.

\section{Appendix A Replication Master Creation}

\section{Photomasks}

A low-cost photomask was designed using AutoCAD software (Autodesk, USA) and sent for printing to MicroLithography Services (Essex, UK) at a resolution of 20,000 dpi.

\section{Glass Slide Preparation}

Glass microscope slides served as the substrate on which patterns were formed to create a replication master. The glass microscope slides (76 mm x $38 \mathrm{~mm}$ x $1.2 \mathrm{~mm}$ ) were obtained from Fisher Scientific (Loughborough, UK). Prior to patterning, the slides were washed in dishwashing liquid and deionised water followed with rinsing under acetone, methanol and isopropyl alcohol. Afterwards the slides were dried with nitrogen gas and then baked for a minimum of 10 minutes on a hot plate set to $110^{\circ} \mathrm{C}$. The slides were then ready for lamination.

\section{Dry Film Lamination}

Rolls of 50 and $75 \mu \mathrm{m}$ thick negative dry film resist (Ordyl AM150 and AM175) were purchased from Elga Europe (Daventry, UK). Operating in a yellow lit room, an A3 office hot roll laminator (GMP Photonex 325) was set to a temperature of $110{ }^{\circ} \mathrm{C}$ and speed 2 . 200 gsm card was cut into several pieces each with dimensions of width $60 \mathrm{~mm}$ and length $220 \mathrm{~mm}$. Each piece of card was then folded in half thus reducing their length to $110 \mathrm{~mm}$. The folded pieces were then fed through the laminator. Ordyl AM150 dry film resist was cut into several pieces with dimensions slightly less than the card pieces, typically $100 \mathrm{~mm}$ long by $50 \mathrm{~mm}$ wide.

The dry film resist (DFR) consisted of two protective layers distinguished by their shiny and matte appearance. Prior to lamination of the glass slides, the DFR is partially laminated to the card. This was done by partially removing the bottom matte protective film by approximately $10 \mathrm{~mm}$, pressing the unprotected laminate to the card causing it to adhere, and then feeding it through the laminator by approximately $5-8 \mathrm{~mm}$ before reversing the laminator.

After pre-adhering the laminate to the card, the remainder of the DFR's bottom protective film was then peeled off completely ensuring the unprotected laminate did not touch the card. A glass slide was then placed on the card taking care to prevent contact between the DFR and slide. During lamination, by hand, the DFR was maintained taut and at angle 
of approximately 30 degrees to the surface of the glass slide and the glass slide held firmly in place. After lamination the glass slide was cut from the card and left to cool for 10 minutes prior to UV exposure.

\section{UV Exposure}

The DFR being a negative photoresist cross-links with exposure to UV light. UV exposure was performed in a custom exposure box fabricated in-house. The box contained a removable infra-red filter and allowed for different intensities of exposure by varying the distance of a shelf containing the sample from the UV light source (see Table A1). The UV light source consisted of a high intensity UV lamp (100 W, $365 \mathrm{~nm}$, Black-Ray B-100AP). This was turned on at least 10 minutes prior to laminating the slides as it required warming up.

Table A1:: UV light intensity versus distance with and without IR filter. Unpublished data courtesy of S. Aghdaei

\begin{tabular}{ccc}
\hline $\begin{array}{c}\text { Distance from Lamp } \\
(\mathrm{mm})\end{array}$ & $\begin{array}{c}\text { Intensity with IR Filter } \\
\left(\mathrm{mW} / \mathrm{cm}^{2}\right)\end{array}$ & $\begin{array}{c}\text { Intensity without IR Filter } \\
\left(\mathrm{mW} / \mathrm{cm}^{2}\right)\end{array}$ \\
\hline 70 & 2.7 & 11.4 \\
125 & 2.2 & 6.4 \\
185 & 1.2 & 4.9 \\
\hline
\end{tabular}

Following the 10 minute cooling period after lamination, a microscope slide was placed laminated side up on a light box shelf on top of a piece of black card. The photomask was then placed on top of the laminated side. Another clean non-laminated microscope glass slide was then placed on top of the photomask. Two small 20 gram masses were then placed on top of the upper glass slide at opposite ends, completing the sandwich. The sandwich was then exposed to UV light for $10 \mathrm{~s}(\approx 115 \mathrm{~mJ}$ dose $)$, with the light box shelf set at a $70 \mathrm{~mm}$ distance from the lamp. After exposure, the laminated slide was then placed on a pre-heated hot plate set to $65^{\circ} \mathrm{C}$ for a duration of 10 minutes. Once baked for 10 minutes, the slide was removed and left to cool to room temperature. The top protective film was then removed from the laminate and the slide developed.

\section{Developing}

In developing the acrylic DFR, a $0.8 \% \mathrm{w} / \mathrm{v}$ aqueous solution of $\mathrm{Na}_{2} \mathrm{CO}_{3}$ (Sigma Aldrich, UK) was prepared fresh. The $\mathrm{Na}_{2} \mathrm{CO}_{3}$ solution was then poured into a Petri dish placed within an ultrasonic cleaner (Ultra 6000, Maplin UK). The laminated slide was first placed laminate side down into the Petri dish and the ultrasonic cleaner switched on. The slide was kept face down for $40 \mathrm{~s}$, then flipped face up for $30 \mathrm{~s}$. The ultrasonic cleaner was then turned off. With the slide still in the Petri dish, the laminate was gently rubbed by hand for $30 \mathrm{~s}$. The slide was then removed and rinsed thoroughly under a water tap. The slide was then visually inspected to determine if further development was required. If further development was required, the process was repeated but at shorter times to avoid overetching. After development the laminated glass slide was cleaned with dishwashing liquid in 
deionized water and then dried with nitrogen. The cleaned slide was then silanized before being used as a replication master.

\section{Silanization}

Silanization was performed under vacuum as described in (Sandison, Zagnoni, AbuHantash, \& Morgan, 2007). Briefly, a drop of trichloro(1H,1H-2H,2H-perfluorooctyl)silane 97\% (Sigma Aldrich, UK) was placed on a coverslip positioned inside a vacuum desiccator. The glass microscope slides were placed within the desiccator positioned around the coverslip. With the application of vacuum the silane drop would bubble creating a vapour, at which point the vacuum pump could be turned off. Whilst leaving the dessicator sealed the slides were coated by the silane vapour. The slides were left in the vapour for 30 minutes before venting the dessicator to atmospheric pressure. Afterwards, the slides were then baked at $120{ }^{\circ} \mathrm{C}$ for 20 minutes.

\section{Appendix B Patterning PDMS}

\section{Patterning Rectangular Profile Channels}

PDMS (Dow Corning Sylgard 184) was purchased from Farnell (Leeds, UK). Preparation of PDMS involved mixing a pre-determined amount of elastomer base and curing agent at a ratio of 10:1 (w/w) in a weighing boat. For reference, the amount of PDMS required to obtain a particular final device thickness can be found in Table B1. The mixed PDMS was then placed in a vacuum dessicator and degassed for 30 minutes to remove air bubbles introduced through mixing. Using the technique described by (O'Neill, Hoo, \& Walker, 2006), aluminium foil was folded and shaped around replication masters creating trays. PDMS was then poured into the trays covering the replication masters. Air bubbles were typically introduced into the PDMS mixture on pouring on to the replication master. These were burst or moved to the periphery of the substrate using a scalpel blade. Persistent bubbles were removed by simply allowing the poured PDMS to stand for several minutes. The poured PDMS was then cured by baking for 1 hour either in a convection oven or hot plate set to $100{ }^{\circ} \mathrm{C}$. After curing the hardened PDMS was then released from the replication master and ready for holes to be punched.

\section{Spin Coating PDMS Films}

PDMS was prepared as described previously. Replication masters were loaded in turn onto a vacuum chuck within a spin coater (Model 4000, Electronic Microsystems Ltd., UK). The spin coater operating parameters were modified from (Zhang, Ferguson, \& Tatic-Lucic, 2004). The spin coater was then set to run at 100 RPM for 15 s allowing approximately 2.5 grams of PDMS to be poured onto the replication master. The spin speed was then ramped up to the final spin speed required (determined from the curve in Figure B1) with an acceleration of $20 \mathrm{RPM} / \mathrm{s}$. The final speed was maintained for $60 \mathrm{~s}$ before stopping. After spinning, the PDMS-covered slides were placed on a hot plate set to $100{ }^{\circ} \mathrm{C}$ and left to cure for 1 hour. Afterwards for the case of bonding, the PDMS was left attached to the glass slides to simplify handling and assembly during bonding. 
Table B1:: PDMS Elastomer Weight and Resulting Layer Thickness

\begin{tabular}{lcr}
\hline $\begin{array}{l}\text { Replication Master Dimensions } \\
(\text { width } \times \text { length }[\mathrm{mm}])\end{array}$ & $\begin{array}{c}\text { Elastomer Base Weight } \\
\text { [grams] }\end{array}$ & $\begin{array}{r}\text { Layer Thickness } \\
{[\mathrm{mm}]}\end{array}$ \\
\hline $25 \times 75$ & 5 & 2.06 \\
$25 \times 75$ & 10 & 4.22 \\
$25 \times 75$ & 15 & 6.94 \\
$35 \times 75$ & 5 & 1.21 \\
$35 \times 75$ & 10 & 2.64 \\
$35 \times 75$ & 15 & 4.5 \\
$50 \times 75$ & 10 & 2.02 \\
$50 \times 75$ & 15 & 3.22 \\
\hline
\end{tabular}

\section{Punching Holes}

Holes were punched in cured PDMS either with a blunt Luer stub or a leather hole punch (Maplin, UK). Listed in Table B2 are recorded measurements of stub and hole punch sizes together with the resulting sized holes in PDMS. After hole punching the PDMS was cleaned by adhering and removing Scotch $\AA$ tape from the PDMS surface. Bonding could then be performed.

Table B2:: Punch and Corresponding Hole Approximate Sizes

\begin{tabular}{lccr}
\hline Type & $\begin{array}{c}\text { Outer Diameter } \\
{[\mathrm{mm}]}\end{array}$ & $\begin{array}{r}\text { Inner Diameter } \\
{[\mathrm{mm}]}\end{array}$ & $\begin{array}{r}\text { Resulting Hole Diameter } \\
\text { in PDMS [mm] }\end{array}$ \\
\hline 20G Stub & 0.91 & 0.51 & 0.40 \\
Punch & & & 1.20 \\
& 4.00 & 2.25 & 1.50 \\
& 4.35 & 2.80 & 1.90 \\
& 4.50 & 3.20 & 2.50 \\
& 5.35 & 3.65 & 2.85 \\
& 5.60 & 4.15 & 3.00 \\
\hline
\end{tabular}

\section{Appendix C \\ Oxygen Plasma Bonding}

A Femto RF oxygen plasma system (Diener Electronic, Nagold, Germany) was used to bond PDMS to PDMS and/or glass. The samples to be bonded were loaded into the oxygen plasma system sample chamber. The sample chamber was pumped down with a vacuum. With an input oxygen flow rate of $70-80 \%$ of $90 \mathrm{sccm} / \mathrm{min}$, the plasma was ignited for 30 s. Afterwards the sample chamber was vented to atmospheric pressure. The samples were 


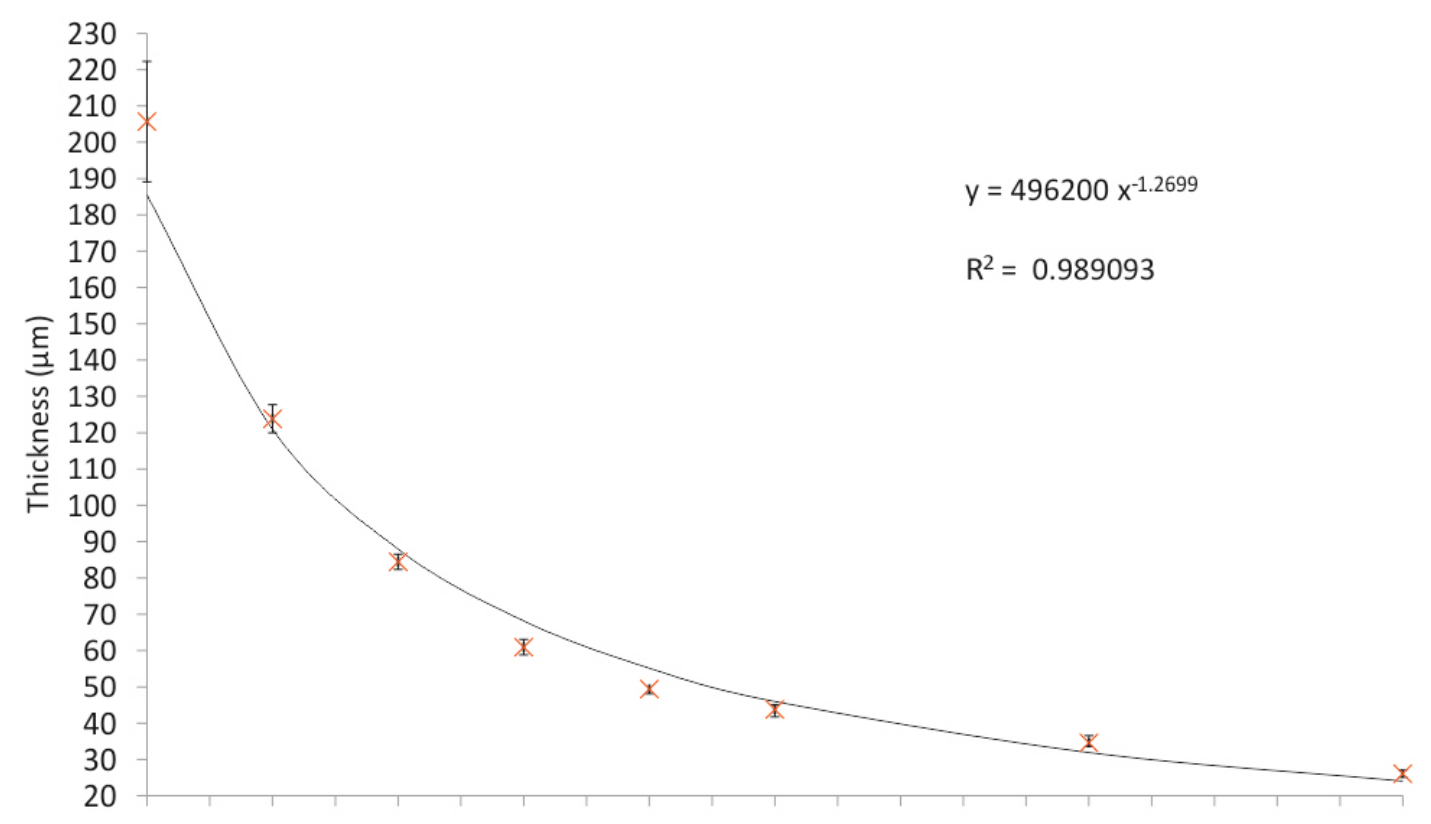

Figure B1. : The thickness of PDMS as a function of spin coating speed.

then removed and brought into contact initiating the bonding process. To finalise bonding, the samples were then baked for 10 minutes on a hot plate set to $75^{\circ} \mathrm{C}$.

\section{Assembling Valved Devices}

Oxygen plasma bonding was used to bond the control and fluidic layers to each other and a glass microscope slide. Alignment of exposed control and fluidic layers was achieved by using deionised water as a lubricant. After oxygen plasma exposure, one to two drops of water from a disposable pipette were placed on the surface of the control layer. The fluidic layer was then brought into contact the control layer, first by contacting one edge and then proceeding with a folding motion to prevent the introduction of air bubbles. Alignment was then performed by sliding the two layers over each other by hand. Once aligned, mild pressure was applied to the two layers by hand for a couple of minutes. The aligned layers were then placed on a hot plate set to $75{ }^{\circ} \mathrm{C}$. Water accumulation in the fluidic channel characterised by a shiny appearance was purged by flowing $\mathrm{N}_{2}(<100 \mathrm{kPa})$ in turn through the inputs and outputs to the channel. Pressure applied by hand to the PDMS layers was maintained whilst purging the water to prevent their separation. Once the water was purged, characterised by a matte appearance, the layers were left to bake for one hour. Afterwards the hot plate temperature was ramped up to $100{ }^{\circ} \mathrm{C}$ and the layers baked for an additional hour. Afterwards the layers were bonded to a glass slide to finalise the valved device assembly. 